\title{
Caesarean scar endometriosis: a case report
}

\section{Fatimazahra Cherrabi, Mounir Moukit*, Jaouad Kouach, Driss Moussaoui Rahali, Mohammed Dehayni}

Department of Obstetrics and Gynecology, Military Training Hospital Mohammed V, Rabat, Morocco

Received: 08 December 2017

Accepted: 08 January 2018

*Correspondence:

Dr. Mounir Moukit,

E-mail: moukitmounir@yahoo.com

Copyright: (c) the author(s), publisher and licensee Medip Academy. This is an open-access article distributed under the terms of the Creative Commons Attribution Non-Commercial License, which permits unrestricted non-commercial use, distribution, and reproduction in any medium, provided the original work is properly cited.

\section{ABSTRACT}

Endometriosis is a common disorder in women of reproductive age but is rarely observed in abdominal scar after caesarean section. The authors report a case of 35-year-old woman referred for a painful lump at caesarean scar. Preoperative diagnosis of caesarean scar endometriosis was made on the basis of clinical examination and radiological imaging and confirmed histologically after large surgical excision of the mass. Increasing awareness of this rare entity among clinicians can help in early diagnosis and appropriate treatment.

Keywords: Caesarean section, Scar endometriosis, Surgical excision

\section{INTRODUCTION}

Endometriosis is a chronic gynaecologic disorder defined by the presence of ectopic functional endometrial tissue outside the uterine cavity. Scar endometriosis is a rare form of endometriosis that is usually confused with other surgical or dermatological lesions leading to delay in diagnosis. Usually, this condition is associated with obstetrical and gynecological interventions although it may occur after other procedures and as denovo. We present a case of cesarean scar endometriosis, diagnosed and managed at a tertiary level centre with a brief literature review. ${ }^{1}$

\section{CASE REPORT}

A 35-year-old multiparous woman was referred in our department for a painful lump at the caesarean scar appeared two years before. She had three caesarean sections previously and the last one being three years ago. She did not have any symptoms suggestive of pelvic endometriosis. Abdominal examinations revealed a tender and firm nodule, measuring $4 \mathrm{~cm} \times 3 \mathrm{~cm}$, near the right angle of her pfannenstiel scar. A differential diagnosis of scar endometriosis, hematoma, or abdominal abscess was made. Ultrasound showed the presence of a superficial well circumscribed hypoechoic mass with moderate vascularity in color Doppler (Figure 1).

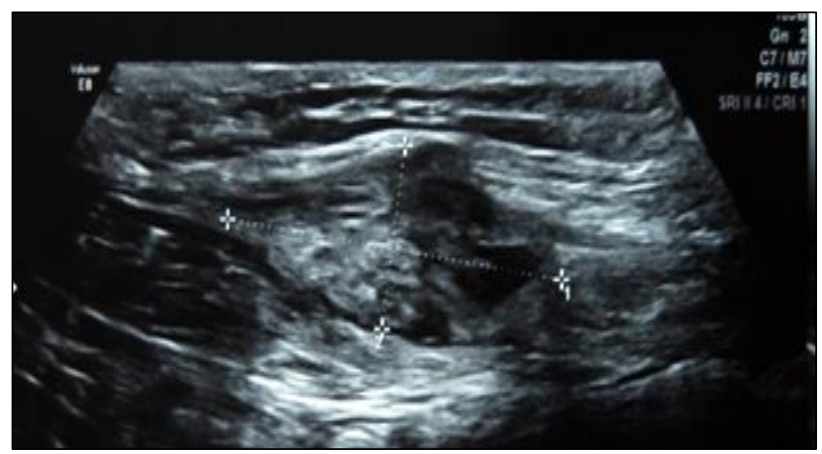

Figure 1: Ultrasound scan of the scar endometriosis.

Magnetic resonance imaging (MRI) objectified an intramuscular lesion of the right rectus abdominis suggestive of endometriosis (Figure 2). There were no 
other lesions of pelvic endometriosis. Under spinal anesthesia, the mass was completely excised with clear margins and the rectus abdominis muscle was repaired using an interrupted absorbable suture (Figure 3). The final pathologic report was endometriosis with no signs of malignancy. Postoperatively, her symptoms have completely resolved, and no recurrence was seen with a follow-up period of 12 months.
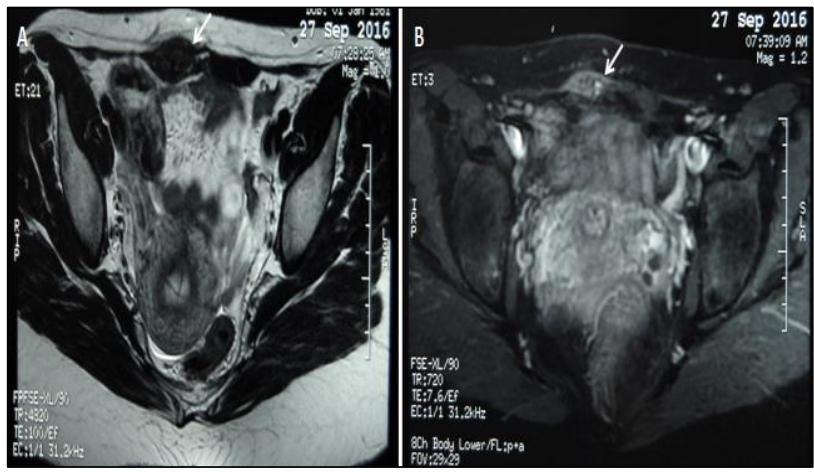

Figure 2: MRI revealing an intramuscular lesion of the right rectus abdominis appearing as a hyposignal on T1-weighted imaging (A/white arrow) and as a heterogeneous hypersignal on $\mathrm{T} 2$-weighted imaging (B/white arrow).

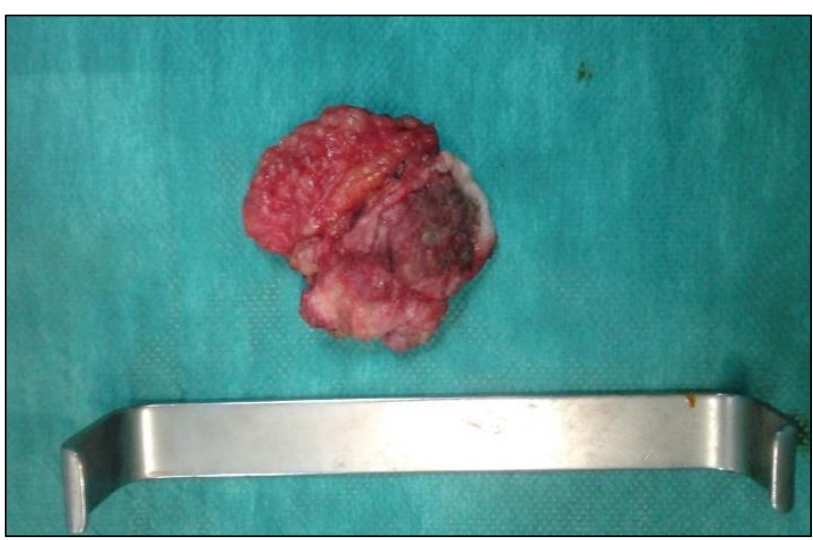

Figure 3: Macroscopic view of the resected specimen.

\section{DISCUSSION}

Scar endometriosis is a very rare entity and its incidence following caesarean section quoted to be $1.96 \%$ in a recent study. ${ }^{1}$ Most studies report that scar endometriosis involves in operations in which uterus is opened. In fact, during surgery, endometrial tissue gets accidentally implanted into the abdominal incision; it grows to form a mass which increases in size during menses and becomes symptomatic. However, it may also occur in operations that need not open the uterus. Douglas and Rotimi reported a case of abdominal incision endometriosis simply after tubal ligation which was caused by tubal mucosa membrane brought into the incision and transformed to the endometrium. ${ }^{2}$ It is interesting to note that Ideyi et al have described a case of spontaneous abdominal wall endometriosis, in absence of previous surgery, highlighting the complex pathogenesis behind the development of scar endometriosis. ${ }^{3}$ The mean period to develop the disease is approximately 12 months, but some cases may take as long as 21 years. ${ }^{2}$ Common symptoms include pain, swelling and swelling during menstruation. Pain in the endometriosis is classically described as cyclical but constant and non-cyclic pattern also have reported as was in our case. ${ }^{4}$

Various imaging techniques are able to give correct diagnosis but lack specificity. According to Hensen et al, ultrasound is the most accessible imaging modality, allowing, along with physical examination - a differential diagnosis with incisional hernia, hematoma, abscess, or sebaceous cyst in most cases. ${ }^{5}$ Computed tomography (CT) or MRI can be used in case of diagnosis doubt and are very helpful in case of involvement of rectus abdominus muscle. The appearances in CT and MRI would depend on the phase of menstrual cycle, the proportion of stromal and glandular elements, amount of bleeding and surrounding inflammatory response. Definitive diagnosis could be made by means of fine needle aspiration cytology (FNAC).

Recent study by Vellido-Cotelo et al reported seventeen cases of endometriosis node in gynecologic scars where a preoperative diagnosis was obtained in $52 \%$ of cases using FNAC and one patient was diagnosed with cancer by this method, which subsequently led to a different therapeutic management. ${ }^{6}$ Theoretically, this procedure has the potential to increased risk of producing new endometriotic implants at the puncture site, especially within concomitant pelvic endometriosis, although this has not been reported. In the present case, clinical examination and imaging features were sufficient to consider preoperatively endometriosis as the cause of the painful lump at the caesarean scar site and there was no need to perform FNAC. According to Gabriel et al, it is impossible to eliminate a mass of $1 \mathrm{~cm}$ entirely with medical treatment. ${ }^{7}$

Gonadotropin-releasing hormone analogues are helpful in alleviating clinical symptoms, but it does not reduce the size of endometrioma and recurrence after the cessation of medication is constant. Consequently, appropriate management is essentially based on large surgical excision of the lesion with reconstruction of damaged tissue. This surgery is effective in preventing recurrence, as well as conversion to malignancy, which - although rare - has been described in a few sporadic cases. ${ }^{8}$

\section{CONCLUSION}

In conclusion, scar endometriosis should be considered in women of reproductive age with pain and/or mass at abdominal scar site following gynecologic or obstetric surgery. Work-up must include both imaging and histopathological findings. Surgical treatment is recommended to excise the lesion completely. 
Funding: No funding sources

Conflict of interest: None declared

Ethical approval: Not required

\section{REFERENCES}

1. Zhang J, Liu X. Clinicopathological features of endometriosis in abdominal wall - clinical analysis of 151 cases. Clin Exp Obstet Gynecol. 2016;43:37983.

2. Douglas C, Rotimi O. Extragenital endometriosis: a clinicopathological review of a Glasgow hospital experience with case illustrations. J Obstet Gynaecol. 2004;24:804-8.

3. Ideyi SC, Schein M, Niazi M, Gerst PH. Spontaneous endometriosis of the abdominal wall. Digestive Surg. 2003;20:246-8.

4. Çöl C, Yılmaz EE. Cesarean scar endometrioma: Case series. World J Clin Cases. 2014;2:133-6.

5. Hensen JH, Vriesman VBAC, Puylaert JB. Abdominal wall endometriosis: clinical presentation and imaging features with emphasis on sonography. AJR. 2006;186:616-20.

6. Vellido-Cotelo R, Muñoz-González JL, Oliver-Pérez MR, Hera-Lázaro CD, Almansa-González C, PérezSagaseta C, et al. Endometriosis node in gynaecologic scars: a study of 17 patients and the diagnostic considerations in clinical experience in tertiary care center. BMC Women's Health 2015;15:13.

7. Gabriel A, Shores JT, Poblete M, Victorio A, Gupta S. Abdominal wall endometrioma: case report and review. Ann Plastic Surg. 2007;58:691-3.

8. Shalin SC, Haws AL, Carter DG, Zarrin-Khameh N. Clear cell adenocarcinoma arising from endometriosis in abdominal wall cesarean section scar: a case report and review of the literature. J Cutaneous Pathol. 2012;39:1035-41.

Cite this article as: Cherrabi F, Moukit M, Kouach J, Rahali DM, Dehayni M. Caesarean scar endometriosis: a case report. Int J Reprod Contracept Obstet Gynecol 2018;7:1221-3. 